\title{
Variabilidad en las indicaciones quirúrgicas de las lesiones intradurales postraumáticas
}

\author{
F. Arikan; J. Sahuquillo; J. Ibáñez; J. Vilalta; M.A. Poca; M. Riveiro*; M.P. Mena**; A. Garnacho* y E. Rubio
}

Servicio de Neurocirugía, Unidad de Cuidados Intensivos de Traumatología*,Unidad de Neurotraumatología**. Hospital Universitario Vall d' Hebron, Barcelona.

Resumen

Introducción. El tratamiento de las lesiones focales intradurales continúa siendo motivo de controversia. Esto es especialmente importante en la decisión de evacuar contusiones cerebrales. Presentamos los resultados de un estudio prospectivo y observacional, cuyo objetivo ha sido analizar la variabilidad en las indicaciones quirúrgicas de lesiones focales intradurales postraumáticas en un servicio de neurocirugía perteneciente a un hospital universitario, dotado de una unidad especializada en neurotraumatología.

Material y métodos. Entre el 1 de mayo y el 31 de diciembre de 2001, se incluyeron 32 pacientes con un traumatismo craneoencefálico (TCE) cerrado, con lesiones focales intradurales. Estos pacientes constituyen la aportación que nuestro centro realizó en el estudio multicéntrico y observacional sobre el manejo de lesiones intradurales dirigido por el European Brain Injury Consortium (EBIC).

Resultados. Las lesiones intradurales de volumen > 25 cc. se evacuaron de forma inmediata al diagnóstico. Nueve de los 13 pacientes con lesiones $<25$ cc. fueron intervenidos de forma diferida debido a la aparición de un deterioro neurológico o al diagnóstico de una hipertensión intracraneal (HIC). Después de la evacuación, la TC de control mostró en todos los casos una clara mejoría en los signos radiológicos de efecto de masa o en el desplazamiento de la línea media.

Conclusiones. A pesar que el reducido número de casos incluidos no nos permiten generalizar las conclusiones, en nuestro centro no se evidencia una variabilidad significativa en las indicaciones quirúrgicas de lesiones $>25$ cc. Sin embargo, existen discrepancias en las indicaciones quirúrgicas de las lesiones con volumen $<25$ cc. Los resultados definitivos del estudio del EBIC permitirán conocer mejor la variabilidad existente en el manejo de este tipo de pacientes.

Recibido: 08-06-04. Aceptado: 08-08-04
PALABRAS CLAVE: Traumatismo craneoencefálico. Lesiones intradurales postraumáticas. Hematomas postraumáticos. Indicaciones quirúrgicas. Variabilidad quirúrgica.

Variability in the surgical indications for posttraumatic intradural lesions

\section{Abstract}

Introduction. The surgical treatment of focal intradural lesions is still a matter of considerable debate. This is especially important in the decision to evacuate brain contusions. We present the results of a prospective observational study in which the main goal was to analyze intracenter variability in the indication for surgery in focal posttraumatic intradural lesions in a department of Neurosurgery of a University Hospital with a specialized neurotrauma unit.

Clinical material and methods. Between May 1 and December 31, 2001, 32 patients with a closed traumatic brain injury and an intradural posttraumatic focal lesion were included. The patients studied were a subgroup included in the European multicenter observational study of the management of intradural lesions conducted under the aegis of the European Brain Injury Consortium (EBIC).

Results. Intradural lesions $>25 \mathrm{cc}$ were immediately evacuated. Nine out of thirteen patients with lesions < 25cc also underwent surgery due to intracranial hypertension or neuroworsening. In all patients in whom lesions were surgically evacuated, the postoperative CT-scan showed neuroradiological improvement of the signs of mass effect or midline shift.

Conclusions. In our center, we found no evidence of significant variability in the indications for surgery in

Abreviaturas. EBIC: European Brain Injury Consortium. GOSE: Glasgow Outcome Scale Extended. HIC: hipertensión intracraneal. PIC: presión intracraneal. PPC: presión de perfusión cerebral. TCDB: Traumatic Coma Data Bank. TCE: traumatismo craneoencefálico. 
intradural lesions of more than 25 cc. However, significant differences were detected among neurosurgeons in the surgical indications for lesions below 25cc. The small sample analyzed precludes generalization of these conclusions. The definitive results of the EBIC study will provide the neurosurgical community with a better understanding of variability in the management of these lesions.

KEY WORDS: Head injuries. Posttraumatic intradural lesions. Posttraumatic hematomas. Surgical indications. Surgical variability.

\section{Introducción}

El tratamiento quirúrgico de las lesiones focales intradurales continúa siendo un tema de debate y que genera controversias en todas las reuniones en las que se aborda el tema del tratamiento de los pacientes neurotraumáticos. La reciente publicación de las guías norteamericanas para el manejo quirúrgico de los pacientes con un TCE ha objetivado la carencia de evidencia científica, al concluir que en el momento actual no existen estudios que nos permitan establecer estándares o guías en el tratamiento quirúrgico de las lesiones intradurales traumáticas ${ }^{1}$. En esta edición, se aconseja como "recomendaciones de nivel tres" el tratamiento quirúrgico en los siguientes grupos de pacientes: 1) pacientes con lesiones intraparenquimatosas y signos de deterioro neurológico progresivo, 2) pacientes con HIC refractaria al tratamiento médico, o signos de efecto masa en la TC cerebral, 3) pacientes con una puntuación en la escala de coma de Glasgow (GCS) entre 6 y 8 con lesiones frontales o temporales de más de $20 \mathrm{cc}$ con desplazamiento de la línea media de más de $5 \mathrm{~mm}$ y/o compresión de las cisternas basales, y 4) pacientes con lesiones de más de $50 \mathrm{cc}$. A pesar de estas recomendaciones la gran variabilidad en las indicaciones quirúrgicas de las lesiones intradurales postraumáticas es una realidad incluso entre los neurocirujanos de un mismo servicio, donde la necesidad de evacuar una determinada lesión suele depender, fundamentalmente, del criterio individual del neurocirujano.

En este estudio presentamos un análisis sobre la variabilidad en los criterios e indicaciones quirúrgicas de las lesiones intradurales traumáticas con efecto de masa, que durante un periodo de 8 meses ingresaron en un hospital universitario, dotado de una unidad especializada en el tratamiento de los pacientes neurotraumáticos. Los 32 pacientes incluidos en este estudio constituyen la aportación que nuestro centro realizó en el segundo estudio epidemiológico multicéntrico y multinacional dirigido por el European Brain Injury Consortium (EBIC), cuya finalidad era obtener información sobre la variabilidad en diferentes países europeos en la utilización de la cirugía como trata- miento de las lesiones traumáticas intradurales.

\section{Material y métodos}

Durante el periodo de tiempo comprendido entre el 1 de Mayo y el 31 de Diciembre se incluyeron todos los pacientes con un TCE, con independencia del GCS, que ingresaron en la Unidad de Neurotraumatología del Hospital Universitario Vall d'Hebron de Barcelona dentro de las primeras 24 horas del traumatismo y en los que la TC cerebral mostró alguna de las siguientes lesiones: 1) lesión intradural cuyo componente hiperdenso superaba los 25 cc, 2) lesiones con un volumen inferior a los $25 \mathrm{cc}$ que presentaban algún signo radiológico indicativo de efecto de masa (desplazamiento de la línea media a nivel del septum pellucidum $=5 \mathrm{~mm}$, compresión u obliteración del tercer ventrículo, compresión u obliteración de la cisterna ambiens ipsilateral, compresión del ventrículo ipsilateral, o dilatación compensadora del ventrículo contralateral). Se incluyeron también aquellos pacientes con lesiones difusas al ingreso, que presentaron una lesión intradural diferida que cumplía los requisitos anteriores.

Se excluyeron todos los pacientes que presentaban alguno de los siguientes criterios: 1) pacientes que no pudieron ser reanimados, 2) lesiones por arma de fuego, 3) hematomas epidurales aislados (no asociados a lesiones intradurales con efecto de masa), 4) hematomas subdurales crónicos que hubieran resangrado, y 5) aquellos pacientes en los que se preveía que no podría realizarse una valoración del resultado final a los 6 meses del traumatismo. El estudio fue aprobado por el Comité de ética del Hospital Universitario Vall d'Hebron, el cual autorizó su realización sin la obtención de consentimiento informado dado el carácter puramente observacional del mismo.

La aportación de nuestro centro en el estudio del EBIC fue de carácter puramente observacional, sin que existiese ningún criterio quirúrgico de obligado cumplimiento en la inclusión o exclusión de los pacientes. Sin embargo, el simple hecho de que los datos de cada centro pudieran ser compartidos con los demás centros participantes y el hecho de saber que los pacientes serían incluidos en el estudio, pudo sesgar de alguna forma las indicaciones quirúrgicas.

\section{Protocolo de tratamiento de los pacientes con un TCE grave en la UCI}

Las medidas generales de actuación que se siguen en nuestra unidad de Neurotraumatología, detalladas en un reciente articulo publicado por Sahuquillo et al. ${ }^{12}$, enfatizan la inmediata evacuación de las lesiones hemáticas focales, así como la prevención de las lesiones isquémicas secundarias. En este protocolo, el objetivo principal es mantener una presión de perfusión cerebral (PPC) por encima de 60 


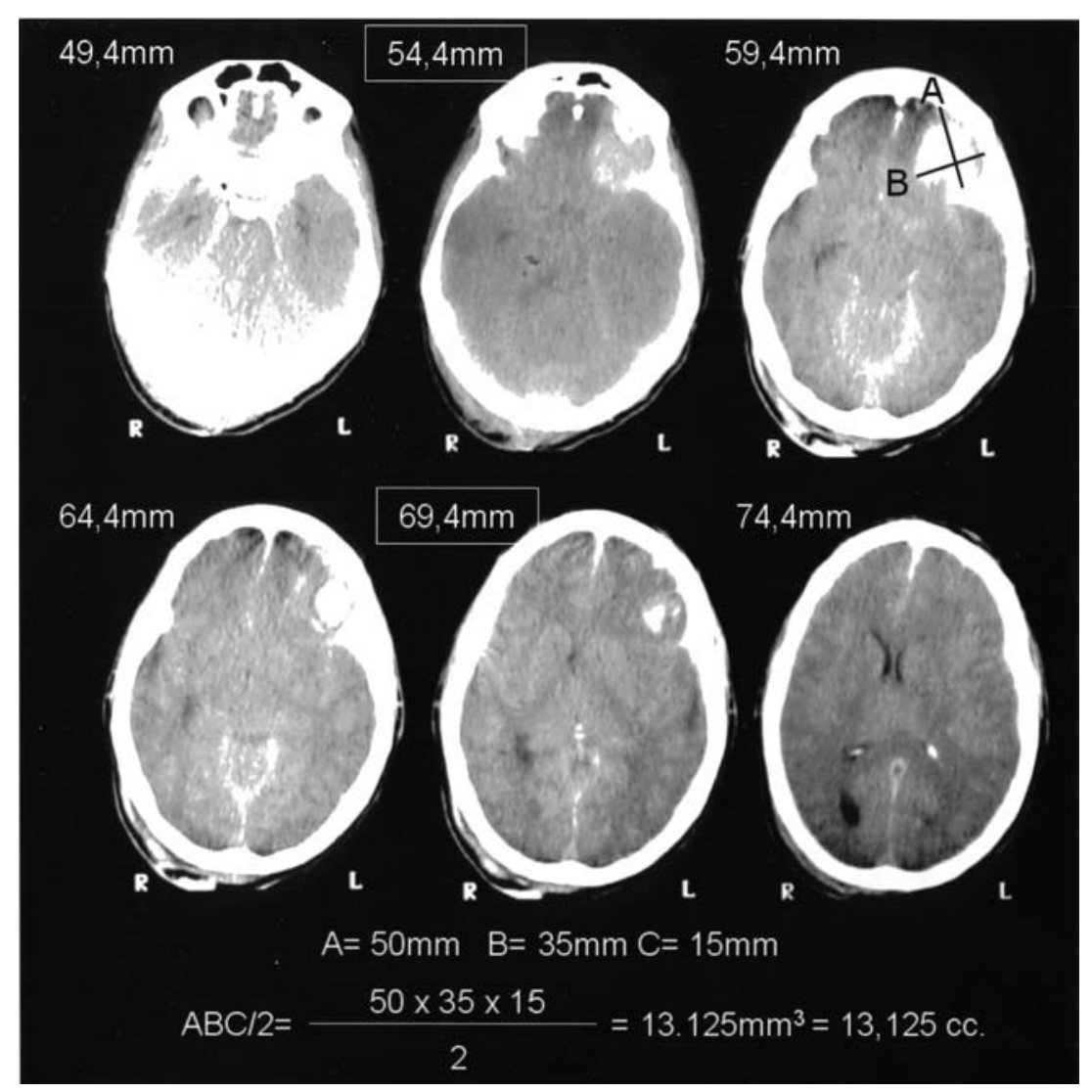

$2005 ; 16: 108-116$

Figura 1. Estimación del volumen de una lesión focal intraparenquimatosa utilizando el método ABC/2. Para utilizar este método se debe seleccionar el corte de la $T C$ donde se observe el área hemorrágica de mayor tamaño. $A$, es la longitud máxima del área hiperdensa (50mm). En el mismo corte se determina el diámetro transverso $B(35 \mathrm{~mm})$. El parámetro $C o$ altura de la lesión, se calcula mediante la substracción de los valores de la "posición de la camilla" del último y primer corte en los cuales se visualice la lesión hiperdensa $(69,4-54,4=15 \mathrm{~mm})$ El producto de $A, B$ y $C$ dividido entre 2 estima el volumen de la lesión. En este ejemplo, el volumen de la lesión es de $13125 \mathrm{~mm}^{3}$ ó 13,125 cc.

$\mathrm{mm} \mathrm{Hg}$ y una presión intracraneal (PIC) inferior o igual a $20 \mathrm{~mm} \mathrm{Hg}$. La PPC se calcula a partir de la diferencia entre la presión arterial media y la PIC, después de enrasar el transductor de presión arterial a nivel del foramen de Monro. Cuando la reposición del volumen es insuficiente para mantener una presión arterial media superior a 90 $\mathrm{mm} \mathrm{Hg}$ se utilizan drogas vasopresoras. Para la sedación y analgesia de estos pacientes se utiliza una combinación de midazolam o propofol y cloruro mórfico. La concentración de hemoglobina se mantiene por encima de $11 \mathrm{~g} / \mathrm{dl}$.

Cuando a pesar de las medidas generales y la sedación y analgesia el paciente presenta una PIC $>20 \mathrm{~mm} \mathrm{Hg}$, el primer paso es descartar la presencia de nuevas lesiones cerebrales, mediante la práctica de una TC de control. En ausencia de nuevas lesiones ocupantes de espacio, se inicia siempre un tratamiento escalonado de la hipertensión intracraneal que incluye, como medidas terapéuticas de "primer nivel", la administración de relajantes musculares (vecuronio), hiperventilación moderada optimizada mediante la monitorización de la saturación venosa de $\mathrm{O}_{2}$ en el bulbo de la yugular y la administración de bolus intermitentes de soluciones hiperosmolares (manitol o sueros salinos hipertónicos al 7,2\%) El coma barbitúrico, la craniectomía descompresiva o la hipotermia moderada son medidas terapéuticas de "segundo nivel", que se utilizan como tratamiento sólo en pacientes con hipertensión

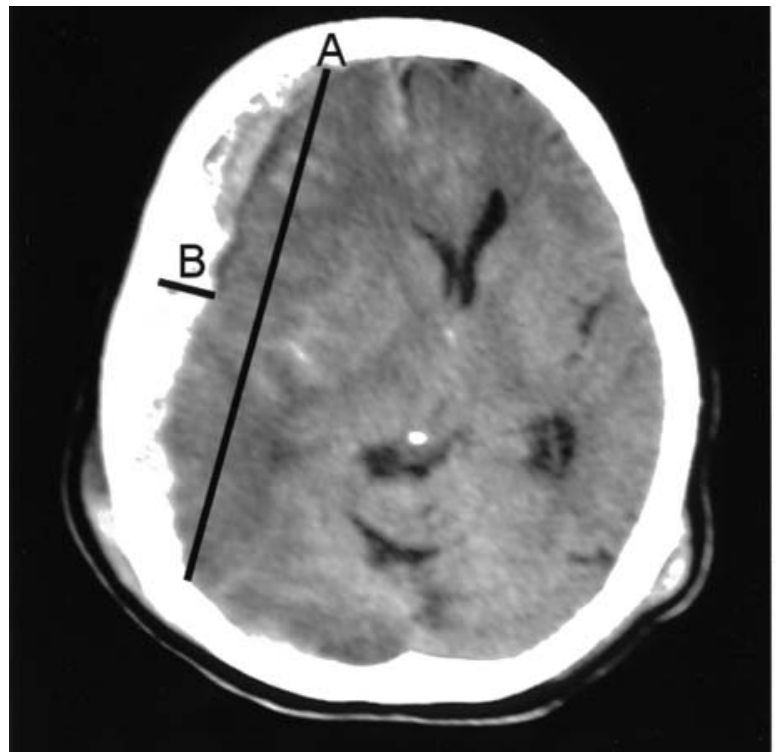

Figura 2. Estimación del volumen en colecciones subdurales. Para ello se selecciona el corte en la TC craneal que muestre la mayor longitud del hematoma, cuya medición desde su extremo anterior al posterior nos dará el valor del parámetro A. El valor B se obtiene en el mismo corte midiendo la máxima medida perpendicular a A. El valor $C$ se obtiene de la misma manera que en las lesiones intraparenquimatosas. 


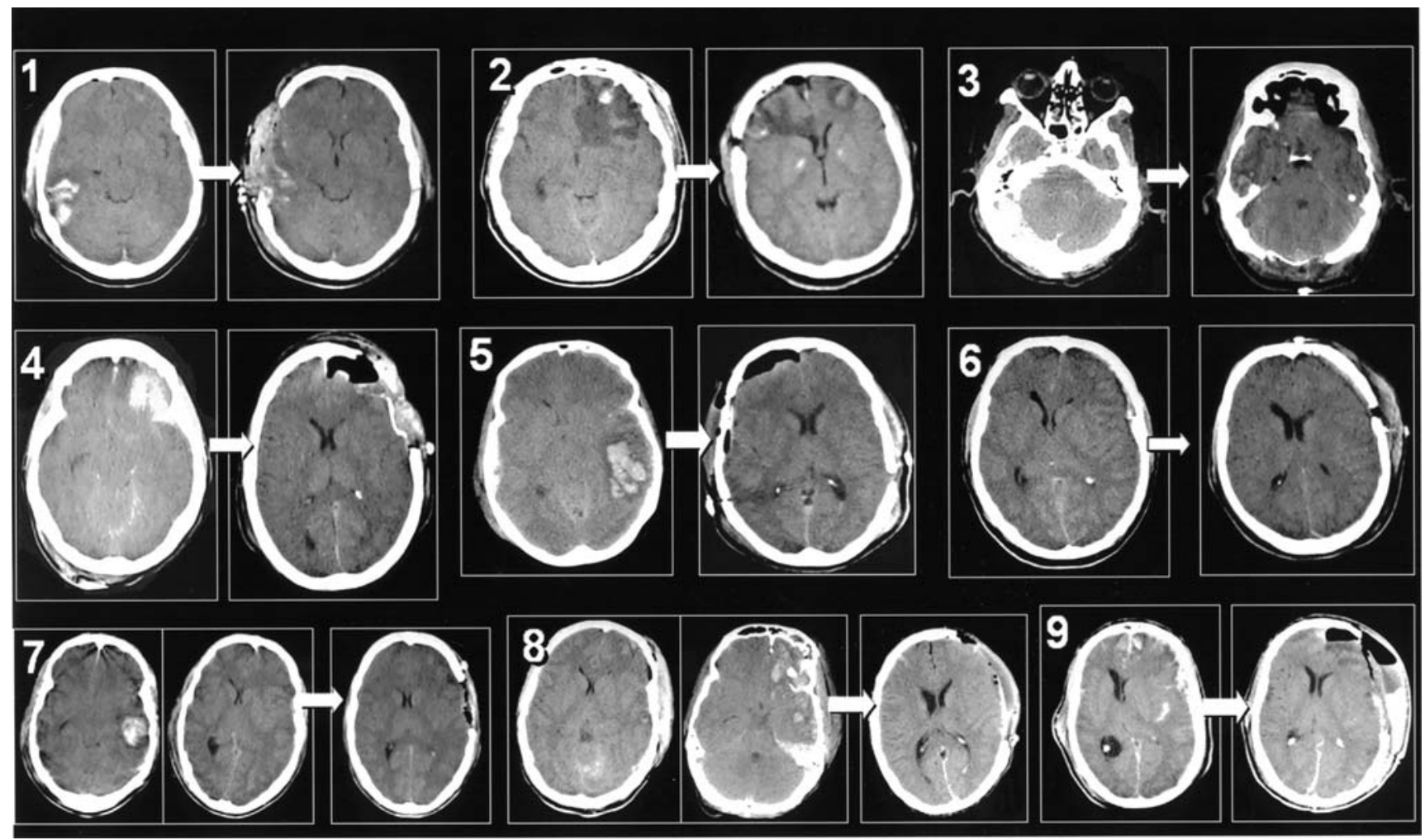

Figura 3. TC pre y post quirúrgicos de los pacientes afectos de lesiones intradurales postraumáticas con volumen inferior a $25 \mathrm{cc}$. Obsérvese como en todos los casos se evidencia una clara mejoría en todos los signos de efecto de masa.

refractaria a las medidas terapéuticas de primer nivel.

El protocolo de actuación incluye la realización de una TC cerebral al ingreso, que se repite a las 6 horas, si la primera exploración se ha realizado de forma inmediata al accidente, entendiéndose como tal si esta primera exploración se realiza en las primeras dos horas desde el accidente. También se realiza una TC cerebral de control cuando existe cualquier deterioro neurológico del paciente, o un aumento, o control inadecuado de la PIC. En situación de estabilidad clínica, la TC cerebral se realiza de forma programada a las 24 y 72 horas del ingreso. La necesidad de realizar exploraciones adicionales de TC se establece de forma individual, de acuerdo con la evolución y el tipo de lesión de cada paciente.

\section{Medición del volumen lesional}

Para la medición del componente hiperdenso de la lesión intradural se siguió el método denominado $\mathrm{ABC} / 2$, fundamentado en el cálculo del volumen de una esfera o un elipsoide ${ }^{8,9,12}$, de acuerdo con la siguiente ecuación: $\mathrm{V}$ $=(4 \pi \mathrm{abc}) / 3$, donde $\mathrm{a}, \mathrm{b}$ y c corresponden a los radios del elipsoide. Para la simplificación del cálculo, se sustituyen los radios por sus respectivos diámetros $(\mathrm{A}, \mathrm{B}$ y $\mathrm{C})$ divididos por 2 y se aproxima el valor de $\pi$ a 3 , lo que permite expresar la fórmula de la siguiente manera: Volumen $=$ $4 / 3(3)(\mathrm{A} / 2 \times \mathrm{B} 2 \times \mathrm{C} / 2)=4(\mathrm{ABC} / 8)=\mathrm{ABC} / 2$.

Para la aplicación de este método en los hematomas intraparenquimatosos se seleccionó el corte de la TC cerebral donde se observó el área hemorrágica de mayor tamaño, siendo A la longitud máxima del área hiperdensa y B el diámetro transversal determinado en el mismo corte. El parámetro $\mathrm{C}$, o altura de la lesión, se calculó mediante la substracción de los valores de la posición de la camilla del último y del primer corte en los cuales se visualizaba la lesión hiperdensa (Fig. 1)

En los hematomas subdurales se seleccionó el corte de máxima longitud del hematoma, medido desde su extremo anterior al posterior (variable A). En este mismo corte se determinó la variable $\mathrm{B}$, que correspondía a la máxima medida perpendicular a $\mathrm{A}$. La variable $\mathrm{C}$ se determinó del mismo modo que en las lesiones intraparenquimatosas (Fig. 2). El volumen de todas las lesiones fue determinado por el mismo observador (F. Arikan).

\section{Protocolo de recogida de variables de interés}

Para la recogida de las variables exigidas se utilizó un sistema centralizado de datos en Internet, proporcionado por el centro coordinador Italiano del EBIC dirigido por 
el Dr. F. Servadei (www.ebic.it). Con este método, cada centro podía introducir las variables de cada paciente de forma directa, accediendo a la Web del EBIC mediante una clave específica. Cada paciente se identificaba por un campo elegido por el centro participante para asegurar su anonimato.

Las variables clínicas principales registradas en todos los pacientes incluyeron la puntuación en la escala de coma de Glasgow (ECG) en el periodo prehospitalario, al ingreso del paciente en nuestro centro y después de la admisión en la UCI de Neurotraumatología. Las variables radiológicas registradas incluyeron el tipo de lesión, evaluada de acuerdo con la clasificación propuesta por Marshall et al. para los estudios del Traumatic Coma Data Bank (TCDB) ${ }^{11}$, la localización de la lesión intradural y los signos radiológicos de efecto de masa. En cada paciente se registró la información radiológica procedente de la $\mathrm{TC}$ al ingreso y de la peor TC practicada durante su curso evolutivo. También se registró la información referente al tratamiento quirúrgico, al tipo de neuromonitorización empleada, y el resultado neurológico del paciente. La valoración neurológica final se realizó a los 6 meses del traumatismo por una neuropsicóloga, de acuerdo con la escala extendida de resultados de Glasgow $(\mathrm{GOSE})^{18}$.

\section{Análisis estadístico}

Las variables recogidas en este estudio se introdujeron en una base de datos específica para su análisis final, mediante el programa estadístico SPSS v.11 (SPSS Inc., Chicago, IL, EEUU). Se realizó un análisis descriptivo de las variables expresándose los resultados mediante la media \pm desviación estándar (DE) para las variables cuantitativas $\mathrm{y}$ en porcentajes para las cualitativas.

\section{Resultados}

\section{Descripción de la serie}

Durante un periodo de 8 meses, nuestro centro incluyó 32 pacientes con lesiones intradurales traumáticas que cumplían los criterios de inclusión exigidos para este estudio. La edad media de los pacientes fue de 47,3 $\pm 21,9$ años (rango 17-82, mediana 44.5). El 75\% de los pacientes (24 casos) fueron varones. De los 32 pacientes incluidos, 18 $(56,2 \%)$ ingresaron directamente en nuestro hospital, mientras que los 14 restantes $(43,8 \%)$ procedían de otro centro hospitalario. Veinte pacientes presentaron un TCE aislado; en siete casos el TCE se asoció a lesiones extracraneales menores; en los otros cinco pacientes el TCE se asoció a lesiones extracraneales mayores, considerando como tales aquellas que requerían por si mismas ingreso hospitalario.

La causa más frecuente del traumatismo fue el acci- dente de tráfico (34\%), seguida de los accidentes domésticos $(22 \%)$. Según la puntuación más representativa de la escala de coma de Glasgow, 15 pacientes presentaron una puntuación igual o inferior a 8 puntos (TCE grave), 10 pacientes una puntuación entre 9 y 13 puntos (TCE moderado), y 7 pacientes entre 14 y 15 puntos (TCE leve) ${ }^{15}$. De acuerdo con la clasificación del TCDB, el tipo de lesión más frecuente en la TC de ingreso fue la lesión difusa tipo II con un $34,4 \%$ (11 pacientes), seguida en un $31,2 \%$ de la lesión tipo VI (diez pacientes), $25 \%$ con lesión tipo difusa IV ( pacientes) y 9,4\% con lesión difusa tipo III (tres pacientes). Las TC cerebrales secuenciales practicadas mostraron un empeoramiento radiológico en 10 pacientes $^{11}$ (Tabla 1 ).

\section{Volumen de las lesiones e indicaciones quirúrgicas}

Al ingreso en nuestro centro, la TC inicial objetivó una lesión intradural de volumen superior a $25 \mathrm{cc}$ en 10 de los 32 pacientes incluidos en el estudio. Seis pacientes con lesiones inicialmente inferiores a $25 \mathrm{cc}$ presentaron un empeoramiento radiológico, con aumento de la lesión por encima del volumen crítico de $25 \mathrm{cc}$. Los 16 pacientes restantes presentaron lesiones intradurales inferiores a 25 cc. Los pacientes de este último subgrupo que presentaron deterioro neurológico o HIC mostraban como característica común la presencia de todos los signos radiológicos de efecto de masa indicados (compresión u obliteración del tercer ventrículo, compresión u obliteración de las cisternas de la base ipsilaterales, compresión del ventrículo ipsilateral y dilatación del ventrículo contralateral).

El $60 \%$ de los pacientes (19 casos) incluidos en este estudio fueron intervenidos quirúrgicamente. Los pacientes con lesiones intradurales con un volumen superior a $25 \mathrm{cc}$ fueron intervenidos de forma inmediata tras su diagnóstico excepto en seis casos, en los que la edad avanzada y la comorbilidad previa hizo que se desestimara el tratamiento quirúrgico. Sin embargo, en nuestro centro existió variabilidad en la indicación quirúrgica de aquellos pacientes en los que a pesar de que el volumen de la lesión era inferior a $25 \mathrm{cc}$ presentaron un deterioro del nivel de conciencia o un mal control de la PIC (13 pacientes) En estos casos la cirugía se indicó a partir de la valoración individual de cada cirujano. (Tabla 2).

Nueve de estos 13 pacientes fueron intervenidos quirúrgicamente. Todos ellos presentaron una mejoría radiológica evidente en el desplazamiento de la línea media y en el resto de signos de efecto de masa (Fig. 3), así como un mejor resultado final en comparación al subgrupo de pacientes que no fueron intervenidos (Tabla 3 ).

Ningún paciente con lesiones intradurales $<25$ cc que no presentara un deterioro neurológico o que mantuviera una presión intracraneal normal fue intervenido. 
Tabla 1

Distribución de los pacientes en función de la clasificación del Traumatic Coma Data Bank (TCDB) según la TC craneal de ingreso y su redistribución de los pacientes que presentaron empeoramiento radiológico. Esta descripción corresponde a las tomografías previas a una actitud quirúrgica

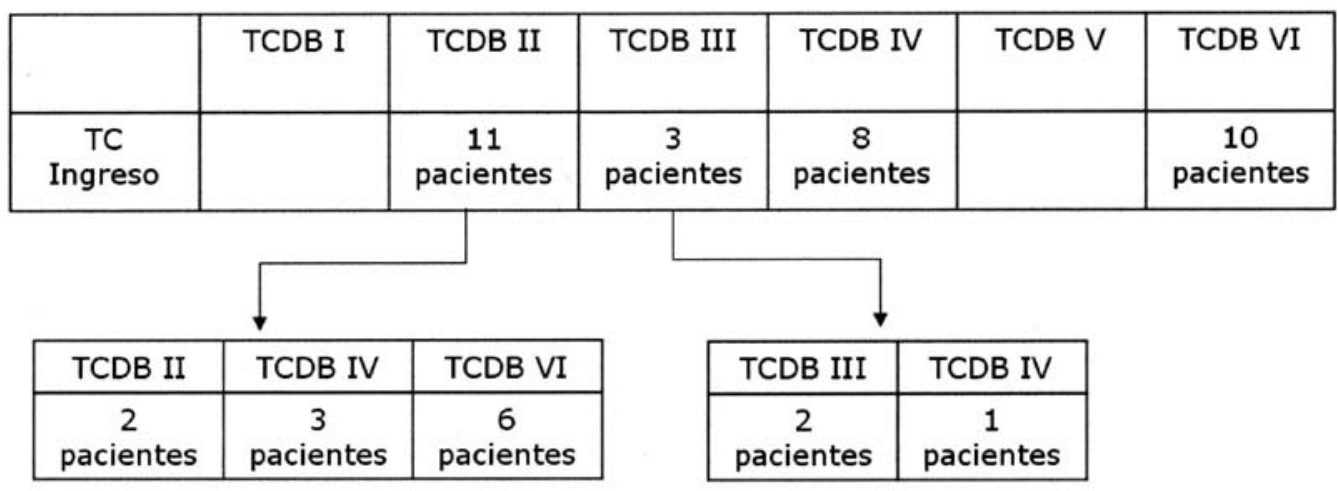

Tabla 2

Actitud quirúrgica según el volumen lesional. Obsérvese como en nuestro centro las lesiones intradurales superiores a 25 cc se intervinieron de forma inmediata al diagnóstico a no ser que la edad avanzada o la comorbilidad previa importante hubiese hecho prever un mal pronóstico. Sin embargo, en las lesiones inferiores a 25 cc es donde existió variabilidad. En los casos que el paciente presentó un deterioro neurológico o una hipertensión intracraneal (HIC) refractaria la decisión de evacuar o no la lesión recavó en la valoración individual de cada cirujano.

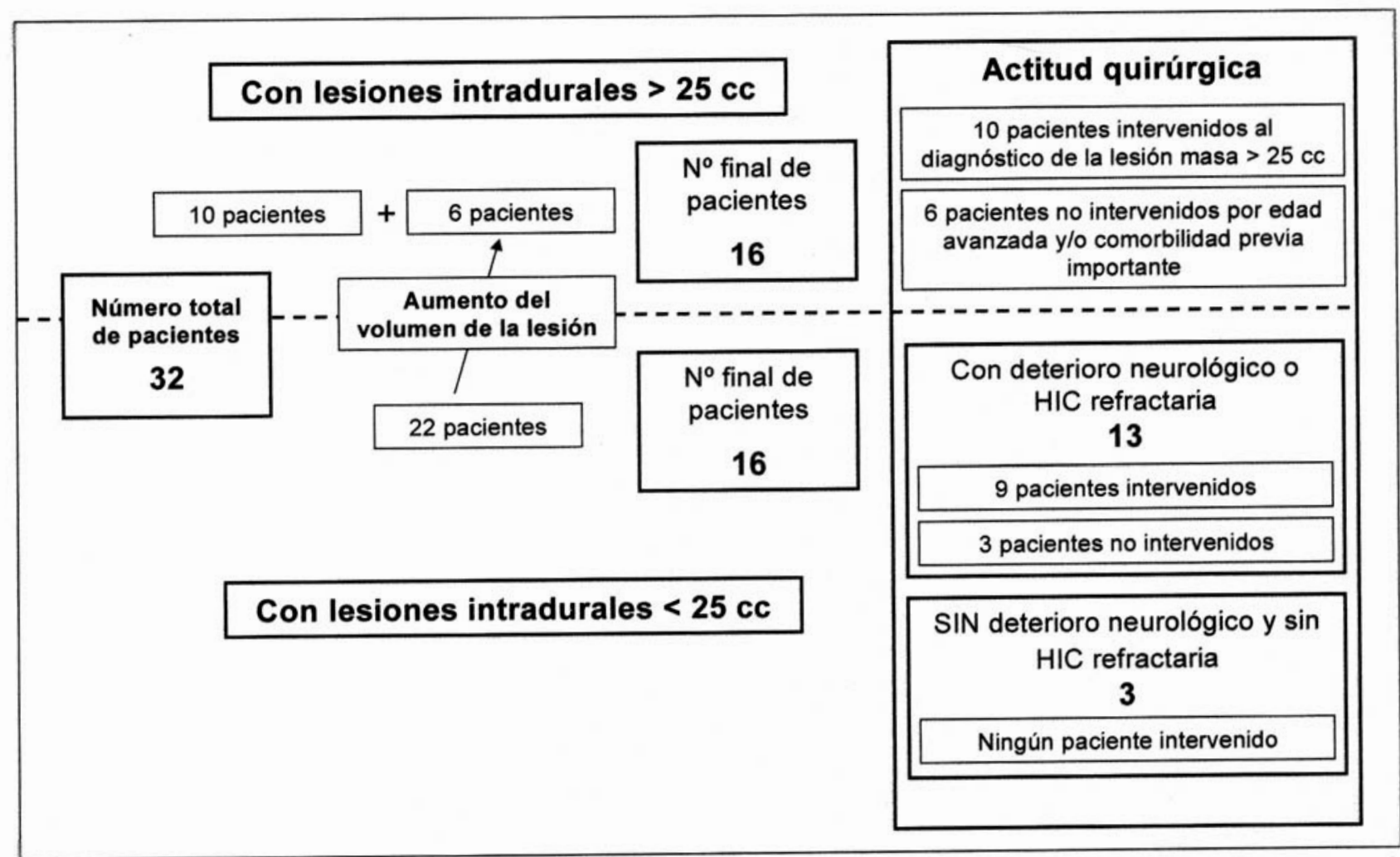


Tabla 3

Relación de los pacientes que presentaron lesiones intradurales con volúmenes inferiores a 25cc según su evolución clínica, intervención quirúrgica y puntuación en la escala de resultados de Glasgow (GOSE) [1=Muerte 2=Estado vegetativo 3=Incapacidad severa, rango bajo 4=Incapacidad severa, rango alto 5=Incapacidad moderada, rango bajo 6=Incapacidad moderada, rango alto 7=Incapacidad leve, rango bajo 8=Incapacidad leve, rango alto] $(\mathrm{HIC}=$ Hipertensión intracraneal)

\begin{tabular}{|c|c|c|c|c|c|}
\hline $\begin{array}{l}\text { Número de } \\
\text { identificación } \\
\text { del paciente }\end{array}$ & $\begin{array}{l}\text { Volumen } \\
\text { de la lesión } \\
\text { intradural } \\
\text { mayor }\end{array}$ & $\begin{array}{c}\text { Desplazamiento } \\
\text { de la línea } \\
\text { media }\end{array}$ & Evolución & $\begin{array}{l}\text { Intervención } \\
\text { quirúrgica }\end{array}$ & GOSE \\
\hline 1 & 15 & 10 & $\begin{array}{c}\text { Deterioro } \\
\text { neurológico }\end{array}$ & SI & 1 \\
\hline 2 & 12 & 9 & HIC & Si & 3 \\
\hline 3 & 10 & 0 & $\begin{array}{c}\text { Deterioro } \\
\text { neurológico }\end{array}$ & Si & 8 \\
\hline 4 & 18 & 6 & HIC & Si & 5 \\
\hline 5 & 23 & 7 & HIC & Si & 7 \\
\hline 6 & 15 & 10 & $\begin{array}{c}\text { Deterioro } \\
\text { neurológico }\end{array}$ & $\mathrm{Si}$ & 8 \\
\hline 7 & 12 & 7 & $\begin{array}{c}\text { Deterioro } \\
\text { neurológico }\end{array}$ & Si & 8 \\
\hline 8 & 5 & 7 & HIC & Si & 3 \\
\hline 9 & 16 & 13 & $\mathrm{HIC}$ & $\mathrm{Si}$ & 3 \\
\hline 10 & 10 & 7 & HIC & No & 1 \\
\hline 11 & 7 & 6 & $\mathrm{HIC}$ & No & 3 \\
\hline 12 & 5 & 2 & $\mathrm{HIC}$ & No & 1 \\
\hline 13 & 10 & 7 & HIC & No & 1 \\
\hline 14 & 11 & 0 & $\begin{array}{l}\text { Buen control } \\
\text { de la PIC }\end{array}$ & No & 3 \\
\hline 15 & 13 & 6 & $\begin{array}{l}\text { Buen control } \\
\text { de la PIC }\end{array}$ & No & 8 \\
\hline 16 & 11 & 0 & $\begin{array}{l}\text { No deterioro } \\
\text { neurológico }\end{array}$ & No & 8 \\
\hline
\end{tabular}

\section{Discusión}

El manejo quirúrgico de los pacientes con lesiones intradurales no está todavía plenamente consensuado. Este hecho queda reflejado en las guías publicadas recientemente por la Brain Trauma Foundation, en las que se objetiva la decepcionante carencia de evidencia científica existente en las indicaciones quirúrgicas de las lesiones intradurales traumáticas ${ }^{1}$. La poca variabilidad existente en nuestro centro en los criterios quirúrgicos de las lesiones intradurales con volumen superior a los $25 \mathrm{cc}$ es un dato remarcable, lo que indica que en nuestro servicio el volumen de la lesión es un factor determinante para la indicación de la cirugía. Este hecho traduce la existencia de un protocolo intrahospitalario consensuado y que concuerda con las diversas guías y recomendaciones publicadas ${ }^{3-5,10}$, las cuales se fundamentan en la clasificación radiológica descrita por Marshall et al. en el análisis de los resultados del Traumatic Coma Data Bank (TCDB) ${ }^{11}$. Sin embargo, debemos recordar que los pacientes de esta serie forman parte de un estudio multicéntrico e internacional y que, de alguna forma, las indicaciones quirúrgicas podrían estar sesgadas por el simple hecho de saber que los pacientes serían incluidos en el estudio y que los datos de cada centro podrían ser de alguna forma evaluados por el resto de cen- 
tros participantes.

En el estudio en que Marshall propone una nueva clasificación radiológica de las lesiones neurotraumáticas, se definió, de una forma aparentemente arbitraria, el concepto de volumen crítico, situándolo por encima de los 25 cc y recomendando este umbral volumétrico para establecer la indicación de evacuación quirúrgica de las lesiones $^{11}$. En nuestro serie, del grupo de pacientes con lesiones intradurales con volumen superior a $25 \mathrm{cc}$, no se intervinieron únicamente aquellos que presentaban una edad avanzada o un estado neurológico que a criterio del neurocirujano hacia prever un mal resultado. A pesar de no existir una contraindicación formal clara en este sentido, la edad y la situación clínica previa del paciente fueron dos factores determinantes para establecer la indicación quirúrgica en cada caso concreto.

Sin embargo, en nuestro centro sí se observó una importante variabilidad en las indicaciones quirúrgicas cuando el volumen no alcanzaba el umbral crítico de los $25 \mathrm{cc}^{11}$. En estos pacientes, la intervención no se realizó en ningún caso por el diagnóstico de lesión intradural, sino de forma diferida, al objetivarse un deterioro neurológico o una HIC refractaria a medidas de primer nivel. En este subgrupo de pacientes con lesiones de menor tamaño, pero con deterioro diferido o hipertensión refractaria, la indicación de cirugía fue realizada de manera individual por el neurocirujano de guardia, apoyándose fundamentalmente en la presencia de signos radiológicos de efecto de masa. No obstante, es importante remarcar que, a pesar del menor volumen de las lesiones focales en los pacientes intervenidos, se evidenció una clara mejoría en la TC postoperatoria, reduciéndose el desplazamiento de la línea media y mejorando el resto de signos radiológicos de efecto masa. Estos pacientes presentaron, además, una mejor evolución clínica respecto a los pacientes que, con HIC o deterioro del nivel de conciencia, no fueron intervenidos.

En la literatura existen discrepancias en las indicaciones quirúrgicas de las lesiones intradurales con volúmenes inferiores a $25 \mathrm{cc}$. Bullock et al. ${ }^{2}$ demostraron que el tamaño de la lesión es tan importante como su localización, mostrando cómo lesiones de gran tamaño localizadas en los lóbulos frontales pueden ser toleradas con un buen resultado neurológico, mientras que lesiones de un tamaño similar en la región temporoparietal requieren una actitud quirúrgica más agresiva. En las lesiones con volúmenes inferiores a $25 \mathrm{cc}$, la conducta más habitual en nuestro centro, y la que refleja la literatura, se limita a evacuar aquéllas que se asocian a signos radiológicos de efecto de masa. También suelen intervenirse los pacientes que se deterioran o que presentan una PIC elevada ${ }^{10}$. Sin embargo, el concepto de efecto de masa en estos casos no es siempre fácil de cuantificar. En general, se considera que la elevación de la PIC $\mathrm{y}$ ciertos hallazgos radiológicos, como un grosor superior a un centímetro en las colecciones subdurales, lesiones intraparenquimatosas de volumen superior a 25-30 cc, un desplazamiento de la línea media superior a $5 \mathrm{~mm}$, la dilatación del ventrículo contralateral al hemisferio de la lesión y la obliteración de las cisternas perimesencefálicas o del tercer ventrículo constituyen signos de riesgo de deterioro neurológico $3,8,10,13,16,17,19,20$.

Al igual que en otros estudios, el nivel de conciencia fue otro aspecto tenido en cuenta en nuestro centro para decidir evacuar ciertas lesiones intradurales de volumen inferior a $25 \mathrm{cc}$. De hecho, algunos autores enfatizan la necesidad de un tratamiento quirúrgico para aquellos pacientes que deterioran de nivel de conciencia o que presentan nuevos déficits neurológicos, con independencia del tamaño de la lesión u otras características radiológicas ${ }^{7,14}$.

Por último, el tercer aspecto que en nuestro centro fue un indicador de tratamiento quirúrgico de las lesiones intradurales inferiores a 25 cc fue la hipertensión intracraneal refractaria. Galbraith y Teasdale ya indicaron en $1981^{6}$ la necesidad de monitorizar la PIC en aquellos pacientes con hematomas intradurales en los que existan dudas sobre la necesidad de evacuación quirúrgica. Sin embargo, es un hecho conocido que en pacientes con lesiones intracerebrales traumáticas en los lóbulos temporales la PIC puede no anticipar el deterioro neurológico o la muerte. Bullock et al. demostraron que en estos pacientes la obliteración de las cisternas de la base o la presencia de un gran edema perilesional eran los factores más importantes para indicar la necesidad de evacuar quirúrgicamente una lesión, incluso cuando el paciente presenta un nivel de conciencia conservado o una PIC normal².

\section{Conclusiones}

El manejo quirúrgico de los pacientes con lesiones intradurales no esta todavía plenamente consensuado. En nuestro centro no se evidenció una variabilidad significativa en el tratamiento de los pacientes con lesiones intradurales con volúmenes superiores a $25 \mathrm{cc}$. Sin embargo, esta falta de arbitrariedad puede reflejar un sesgo de selección, debido a la mera participación en un estudio internacional. Las recomendaciones que aparecen en la literatura sugieren el tratamiento quirúrgico en estos pacientes. En las lesiones intradurales de volumen inferior a $25 \mathrm{cc}$, la indicación quirúrgica fue variable. Los resultados obtenidos en nuestro centro plantean la necesidad de protocolizar esta patología, recomendando intervenir a todos aquellos pacientes con lesiones intradurales inferiores a $25 \mathrm{cc}$ que muestren signos radiológicos de efecto de masa (compresión u obliteración del tercer ventrículo, compresión u obliteración de las cisternas de la base ipsilaterales, compresión del ventrículo ipsilateral, o dilatación del ventrículo contralateral) y/o que presenten además HIC refractaria o deterioro del nivel de 
conciencia. Sin embargo, el reducido tamaño de la muestra no nos permite extrapolar nuestras conclusiones obtenidas a otros centros, por lo que debemos esperar a la publicación de los resultados definitivos del estudio EBIC para conocer mejor la variabilidad en el uso de la cirugía en este tipo de lesiones.

\section{Agradecimientos}

Agradecemos al Executive Comittee del European Brain Injury Consortium, permitirnos la publicación de este grupo de pacientes antes de la publicación definitiva del estudio completo.

Este estudio ha sido financiado en parte por la Red Temática de Investigación Cooperativa de Enfermedades Neurológicas (RED CIEN) del Fondo de Investigaciones Sanitarias $(\mathrm{C} 3 / 06)$.

\section{Bibliografía}

1. Bullock, M. R., Chesnut, R., Ghajar, J., et al: Guidelines for the Surgical Management of Traumatic Brain Injury. http://www2.braintrauma.org/guidelines/downloads/btf_guidelines_surgic al.pdf?BrainTrauma_Session=bfe $7 d 76$ debled 75edc784bac442b89e6 . 2002. Ref Type: Electronic Citation.

2. Bullock, R., Golek, J., Blake, G.: Traumatic intracerebral hematoma--which patients should undergo surgical evacuation? CT scan features and ICP monitoring as a basis for decision making. Surg.Neurol. 1989; 32: 181-187.

3. D'Avella, D., Brambilla, G.L., Delfini, R., et al,: Guidelines for the treatment of adults with severe head trauma (part III). Criteria for surgical treatment. J. Neurosurg. Sci. 2000; 44: 19-24.

4. D'Avella, D., Cacciola, F., Angileri, F.F., et al.: Traumatic intracerebellar hemorrhagic contusions and hematomas. J. Neurosurg. Sci. 2001; 45: 29-37.

5. D'Avella, D., Servadei, F., Scerrati, M., et al.: Traumatic intracerebellar hemorrhage: clinicoradiological analysis of 81 patients. Neurosurgery. 2002; 50: 16-25.

6. Gallbraith, S., Teasdale, G.: Predicting the need for operation in the patient with an occult traumatic intracranial hematoma. J. Neurosurg. 1981; 55: 75-81.

7. Kakarieka, A., Braakman, R., Schakel, E.H.: [Classification of head injuries based on computerized tomography: prognostic value]. Neurologia. 1995; 10: 159-161.

8. Kasner, S.E.: Geometry and subdural hematoma volume. Stroke. 1999; 30: 188.

9. Kothari, R.U., Brott, T., Broderick, J.P., et al.: The ABCs of measuring intracerebral hemorrhage volumes. Stroke. 1996; 27: 1304-1305.

10. Maas, A.I., Dearden, M., Teasdale, G.M., et al.: EBICguidelines for management of severe head injury in adults. European Brain Injury Consortium. Acta Neurochir. (Wien.). 1997; 139: 286-294.

11. Marshall, L.W., Eisenberg, H.M., Jane, J.A., et al.: A new classification of head injury based on computerized tomography. J. Neurosurg. 1991; 75: S14-S20.

12. Sahuquillo, J., Biestro, A., Mena, M.P., et al.: [First tier measures in the treatment of intracranial hypertension in the patient with severe craniocerebral trauma. Proposal and justification of a protocol]. Neurocirugía. 2002; 13: 78-100.

13. Seelig, J.M., Becker, D.P., Miller, J.D., et al.: Traumatic acute subdural hematoma: major mortality reduction in comatose patients treated within four hours. N. Engl. J. Med. 1981; 304: 1511-1518.

14. Servadei, F., Murray, G.D., Penny, K., et al.: The value of the "worst" computed tomographic scan in clinical studies of moderate and severe head injury. European Brain Injury Consortium. Neurosurgery. 2000; 46: 70-75.

15. Stein, S.C.: Minor head injury: 13 is an unlucky number. J. Trauma. 2001; 50: 759-760.

16. Stone, J.L., Rifai, M.H., Sugar, O., et al.: Subdural hematomas. I. Acute subdural hematoma: progress in definition, clinical pathology, and therapy. Surg. Neurol. 1983; 19: 216-231.

17. Wilberger, J.E., Jr., Harris, M., Diamond, D.L.: Acute subdural hematoma: morbidity, mortality, and operative timing. J. Neurosurg. 1991; 74: 212-218.

18. Wilson, J.T., Pettigrew, L.E., Teasdale, G.M.: Structured interviews for the Glasgow Outcome Scale and the extended Glasgow Outcome Scale: guidelines for their use. J. Neurotrauma. 1998; 15: 573-585.

19. Wong, CW.: Criteria for conservative treatment of supratentorial acutesubdural haematomas. Acta Neurochir. (Wien.). 1995; 135: 38-43.

20. Zumkeller, M., Behrmann, R., Heissler, H.E., et al.: Computed tomographic criteria and survival rate for patients with acute subdural hematoma. Neurosurgery. 1996; 39: 708712 .

Arikan, F.; Sahuquillo, J.; Ibáñez, J.; Vilalta, J.; Poca, M.A.; Riveiro, M.; Mena, M.P.; Garnacho, A.; Rubio, E.: Variabilidad en las indicaciones quirúrgicas de las lesiones intradurales postraumáticas. Neurocirugía 2005; 16: 108-116.

Correspondencia postal: Fuat Arikan Abelló. C/ Mora de Ebro 65-67 esc. A 2 ${ }^{\mathrm{o}}-4^{\mathrm{a}}$. 08023 Barcelona. 